Vol. 4, $n^{\circ} 1 \mid 2000$

Varia

\title{
Justice, infrajustice, parajustice et extra justice dans la France d'Ancien Régime
}

\section{Benoît Garnot}

\section{(2) OpenEdition \\ Journals}

Édition électronique

URL : https://journals.openedition.org/chs/855

DOI : $10.4000 /$ chs.855

ISSN : 1663-4837

Éditeur

Librairie Droz

Édition imprimée

Date de publication : 1 janvier 2000

Pagination : 103-120

ISBN : 2-600-00433-5

ISSN : 1422-0857

\section{Référence électronique}

Benoît Garnot, « Justice, infrajustice, parajustice et extra justice dans la France d'Ancien Régime », Crime, Histoire \& Sociétés / Crime, History \& Societies [En ligne], Vol. 4, $\mathrm{n}^{\circ} 1$ | 2000, mis en ligne le 02 avril 2009, consulté le 23 mars 2022. URL : http://journals.openedition.org/chs/855 ; DOI : https://doi.org/ $10.4000 /$ chs. 855 


\title{
Justice, infrajustice, parajustice et extrajustice dans la France d'Ancien Régime
}

\author{
Benoît Garnot'
}

\begin{abstract}
Dans la France d'Ancien Régime, une proportion importante du traitement de la criminalité n'est pas assurée par la justice, mais par l'infrajustice. Mais si l'attention portée à l'infrajustice constitue un progrès essentiel de la recherche, on peut craindre qu'elle ne débouche sur une nouvelle «illusion historiographique»: on a trop tendance à exagérer sa fréquence et à en faire une sorte de panacée, oubliant ainsi qu'une part importante de la criminalité échappe au traitement non seulement judiciaire, mais aussi infrajudiciaire. Cette erreur découle d'une définition à la fois imprécise et excessive de l'infrajustice, trop souvent confondue avec le traitement social de tous les écarts aux normes. Cet article cherche donc à préciser les limites de l'influence de la justice dans le traitement des conflits, à définir précisément l'infrajustice et ses modalités, et à insister sur les comportements qui n'appartiennent ni à la justice ni à l'infrajustice, que je propose de regrouper pour les uns sous le terme de "parajustice», pour les autres sous celui d'"extrajustice».
\end{abstract}

In France, under the Old Régime, a high proportion of crimes did not come before the courts but were dealt on an infrajudicial level. But, although interest in infrajustice constitutes a crucial step for research, the fear that it may turn out to be yet another "historiographical illusion» is altogether legitimate: there is a tendency to exaggerate the frequency of the phenomenon and to make it a sort of panacea, thus forgetting that a great amount of criminal behavior not only is not dealt with by the judicial system, but is missed by the infrajudicial system as well. This error stems from a not only vague but excessive definition of infrajustice, which is too often confused with the social treatment of all abnormal behavior. The present article therefore seeks to specify the limits of the justice system in dealing with conflicts, to clearly define what constitutes infrajustice and its modalities, and to underscore the behaviors that do not belong to either the justice or the infrajustice system, which I would suggest putting under the heading, for the first, of "parajustice» and, for the second, "extrajustice».

I Benoît Garnot est professeur d'histoire moderne à l'université de Bourgogne (Dijon-France), où il dirige le Centre d'études historiques sur la criminalité et les déviances (UMR CNRS 5605). Spécialiste de l'histoire des comportements et des mentalités, il a publié une vingtaine d'ouvrages, parmi lesquels: Un crime conjugal au XVIIT siècle. L'affaire Boiveau, Paris, 1993; Vivre en Bourgogne au XVIII siècle, Dijon, Éditions universitaires de Dijon, 1996; Crime et justice aux XVII et XVIIF siècles, Paris, Imago, 2000. Il a également dirigé plusieurs ouvrages collectifs, dont récemment: La petite délinquance du Moyen Age à l'époque contemporaine, Dijon, Éditions universitaires de Dijon, 1998. Il développe et dirige actuellement des travaux fondés sur l'exploitation des archives des justices seigneuriales bourguignonnes d'Ancien Régime. 
Ce qui vient à la connaissance de la justice d'Ancien Régime ne constitue qu'une partie, la minorité probablement, des crimes réellement commis. Comment croire, par exemple, qu'une province comme l'Anjou n'ait connu au cours du XVIII ${ }^{e}$ siècle qu'une dizaine de viols, ainsi que le laissent supposer les archives judiciaires ${ }^{2}$, ou admettre que dans le ressort du parlement de Toulouse la proportion des appels criminels à la Tournelle du parlement, même s'ils progressent au cours du siècle d'une à deux centaines par an, révèle un taux de criminalité apparente constamment inférieur à un pour dix mille habitants ${ }^{3}$ ? Sans nul doute, une proportion importante du traitement de la criminalité n'est pas assurée par la justice, mais par l'infrajustice, ce dont témoignent, outre quelques sources ponctuelles (mémoires, correspondances, etc.), les archives judiciaires elles-mêmes, qui font allusion incidemment, au fil des témoignages et des interrogatoires, à des pratiques infrajudiciaires, ainsi que les nombreux accords passés devant notaires entre des parties ${ }^{4}$. Sur ce point, la cause paraît être maintenant entendue chez la plupart des historiens, non seulement de la France moderne ${ }^{5}$, mais aussi des autres pays ${ }^{6}$.

Pourtant, si l'attention portée à l'infrajustice constitue un progrès essentiel de la recherche, on peut craindre qu'elle ne débouche sur une nouvelle «illusion historiographique », après celle qui avait, en son temps, fait à tort des archives de la répression judiciaire le miroir de la criminalité réelle ${ }^{7}$ : si la réalité et les modalités de l'infrajustice commencent à être relativement bien connues, on a trop tendance à exagérer sa fréquence et à en faire une sorte de panacée, oubliant ainsi qu'une part importante de la criminalité échappe au traitement non seulement judiciaire, mais aussi infrajudiciaire. Cette erreur découle d'une définition à la fois imprécise et excessive de l'infrajustice, trop souvent confondue avec le traitement social de tous les écarts aux normes qui ne trouvent pas de solution devant les tribunaux, quel que soit le mode de ce traitement: d'où un concept beaucoup trop vague pour être vraiment opératoire ${ }^{8}$. Il s'agira donc ici de préciser d'abord les limites de l'influence de la justice dans le traitement des conflits au sein de la France d'Ancien Régime, de définir ensuite précisément l'infrajustice et ses modalités, enfin d'insister sur les comportements qui n'appartiennent ni à la justice ni à l'infrajustice, que je propose de regrouper pour les uns sous le terme de "parajustice», pour les autres sous celui d'«extrajustice».

\section{1. - LA JUSTICE REFUSÉE?}

La connaissance d'une grande partie, la plus grande peut-être, des affaires criminelles échappe à la justice, leur traitement aussi par conséquent. Cette réalité est davantage la conséquence des choix effectués par la population que des faiblesses, qui sont réelles, de la justice et de la police.

Archives nationales, Inventaire 450.

Castan, N. et Y. (1982, p. 361).

Soman (1982).

En particulier grâce à Castan, N. (1977 et 1980); Castan, Y. (1979).

Voir notamment Lenman, Parker (1980); Shoemaker (1991); Mantecon (1997).

Garnot (1989).

8 Comme dans Garnot (1996, p. 467-471). 


\section{A. Les réticences envers la justice}

Si la population préfere si souvent ne pas faire appel à la justice, c'est pour des raisons qui relèvent de critères qui peuvent être généraux, spécifiques ou personnels.

Les raisons générales du refus du recours à la justice sont diverses et variées, et elles peuvent se cumuler. Elles découlent d'abord de la peur qu'inspire celle-ci avec ses châtiments parfois terrifiants, surtout aux $\mathrm{XVI}^{\mathrm{e}}$ et XVII ${ }^{\mathrm{e}}$ siècles lorsque s'affirme le «temps des supplices ${ }^{9}$, mais aussi avec les fortes amendes qu'elle inflige aux plaignants déboutés comme aux coupables condamnés, sans parler des frais de fonctionnement que les parties doivent acquitter; de sorte que si un coupable a évidemment tout intérêt à éviter la justice, c'est aussi le cas pour une victime si elle veut faire l'économie d'une procédure qui peut être longue, coûteuse et parfois incertaine: les «arbitrages» du Carcassonnais, du Limousin et du Narbonnais, par exemple, actes notariés où les deux parties déclarent qu'elles renoncent mutuellement à une action judiciaire, précisent très nettement qu'elles le font à cause des frais qui s'ensuivraient ${ }^{10}$. Les raisons du refus de la justice peuvent tenir aussi à la nécessité de préserver l'honneur et la réputation ${ }^{11}$, comme dans les affaires de viol, où le passage devant une cour de justice risque d'entraîner, avec la publicité, le déshonneur pour la victime et pour sa famille. Elles s'expliquent également par cette faiblesse essentielle de la procédure inquisitoire qu'est, aux yeux de la population, son exclusion d'une grande partie du processus judiciaire, d'où l'assimilation fréquente par elle de la justice à un pouvoir abstrait, d'inspiration tyrannique, au langage obscur et inacceptable: il ne s'agit pas vraiment d'une contestation de l'ordre social, lequel est généralement admis par tous (ou presque tous), mais à l'intérieur de ce cadre globalement accepté, les gens entendent maintenir le domaine de leur autonomie et placer une part importante de leur vie hors du champ des surveillances, bref, arranger leurs affaires entre eux, ce dont témoignent aussi dans les affaires quand même portées en justice la fréquente inertie («déclare ne rien savoir des faits enquis») ou la nette réticence (le refus d'aller déposer) de nombreux témoins, toutes attitudes qui enlèvent au procès criminel concerné toute chance de succès. Entrent certainement aussi en jeu des raisons d'ordre religieux : le devoir de paix s'impose au chrétien et l'offensé peut préférer accorder le pardon à son agresseur plutôt que de le traîner en justice ${ }^{12}$. Toutes ces raisons reviennent finalement à l'existence, au sein de la population, d'un système de valeurs différent de celui exprimé par la législation.

Tels sont les critères généraux qui expliquent le refus ou le choix du recours à la justice; mais au cas par cas, entrent aussi en jeu des critères spécifiques à la nature des coupables et de leur faute: on peut en distinguer trois. Le premier critère est social, c'est celui de la non-intégration du (supposé) coupable: il ne relève donc pas de la nature du délit, mais de celle du délinquant. La marque principale de cette situation est la non-appartenance à la communauté, par la naissance ou par le mariage, ou encore par le style de vie; les fous, les voleurs, même certains meurtriers, s'ils sont natifs du lieu ou ont fini par être adoptés par les habitants, sont

$9 \quad$ Muchembled (1992).

10 Larguier (1996, p. 407).

"Sur l'honneur, voir Muchembled (1989, p. 43, 253 s.).

12 Voir notamment l'exemple des pardons catalans dans Larguier (1996). 
considérés comme appartenant à la communauté et de ce fait sont rarement livrés à la justice, sauf excès considérés comme intolérables (et encore!). Mais envers les "étrangers », résidents depuis peu ou de passage (notamment les vagabonds dans les campagnes), l'appel à la maréchaussée ou à la justice est quasi systématique en cas de crime réel ou supposé, et il semble même qu'ils fournissent aisément des coupables de substitution à la place de tel ou tel individu intégré dans la communauté ${ }^{13}$.

Le second critère, qui concerne les habitants intégrés, tient à la façon dont la faute a été commise, c'est-à-dire au respect de règles de comportement admises par tous, en particulier celles qui relèvent de l'honneur. Pour les homicides, par exemple, les communautés ne portent en justice ni ceux qui n'ont pas été prémédités, en particulier lorsqu'ils sont la conséquence d'un coup mal contrôlé lors d'une rixe (une maladresse, en quelque sorte), ni ceux dont les motifs apparaissent comme honorables, surtout quand il s'agissait pour le coupable de défendre son honneur bafoué par la victime (dans ce cas, l'exercice de la violence, homicide ou tentative d'homicide compris, est considéré par la population non seulement comme parfaitement légitime, mais comme nécessaire, et bénéficie d'un évident soutien moral); il en va de même dans les affaires d'intérêts, à condition d'être dans son droit, et pour les réactions à des injustices. En revanche, les communautés n'admettent pas, donc portent souvent en justice, les homicides prémédités et «déloyaux » (les guetapens, les meurtres par tueurs à gages...), qui leur paraissent transgresser les règles de la loyauté et de l'honneur d'après lesquelles chacun doit avoir sa chance dans un combat ${ }^{14}$.

Le troisième critère relève de ce qu'on pourrait appeler un «seuil de tolérance», c'est-à-dire le danger excessif qu'un individu paraît faire courir à l'ensemble de la communauté. Si les auteurs de crimes et de larcins ne portent pas, a priori, une tare indélébile aux yeux des villageois, c'est à condition de ne pas exagérer, donc de ne pas se montrer coupables d'exactions multipliées, quelles qu'elles soient, qui finissent par les rendre insupportables. On constate souvent la longue patience et la résignation des victimes, jusqu'à ce qu' une infraction de trop fasse franchir le seuil du recours à la justice; d'où l'ouverture de l'enquête, au cours de laquelle les langues se délient peu à peu et se révèle une multitude d'infractions. Mais c'est vraiment le dernier recours, après une longue tolérance (décidée ou imposée), ou après que d'autres solutions ont éventuellement été tentées et ont échouée ${ }^{15}$.

Restent les raisons personnelles du refus du recours à la justice, lesquelles recoupent fréquemment des réalités sociales. Dans un milieu donné, l'unanimité sur les conduites à adopter n'est pas forcément la règle, en effet, et il peut arriver que certains la transgressent pour porter une affaire en justice. Il semble que ce soit surtout le cas des notables, en particulier au XVIII ${ }^{\mathrm{e}}$ siècle, mais seulement dans certaines circonstances. L'institution judiciaire peut ainsi être utilisée par certains éléments du monde paysan qui pensent en tirer avantage pour conforter leur puissance économique et sociale; on le constate par exemple chez des gros fermiers d'île-de-France dans les conflits qu'ils peuvent avoir avec les ouvriers agricoles. Il arrive aussi, surtout dans les affaires de sang, que les «élites» locales, en particulier lorsque leurs

\footnotetext{
13 Garnot (1999).

14 Pour un exemple précis, voir Garnot (1993).

15 Voir par exemple Figueira (1995), qui constate que les voleurs poursuivis en justice en étaient tous au moins à leur dixième larcin connu de tous...
} 
liens avec la communauté sont ténus ${ }^{16}$ ou lorsqu'elles sont en conflit avec elle pour telle ou telle raison, estiment que la loi doit être appliquée et pour cela déposent une plainte. Ce type de réaction est sans doute plus fréquent au XVIII ${ }^{\mathrm{e}}$ siècle, surtout dans sa seconde moitié, que pendant les deux siècles précédents, ce qui prouverait une évolution des mentalités dans au moins une frange de la population, ce qu'on pourrait appeler une «acculturation judiciaire».

\section{B. Les faiblesses de la justice}

Face à la réticence (c'est le moins que l'on puisse dire) de la population à faire appel à elles, la justice et la police ont des moyens de connaissance et d'action limités, même s'ils augmentent au cours de la période: il n'est pas question de les présenter ici en détail, mais seulement de rappeler et de mettre en perspective quelques réalités. Ainsi dans les campagnes, pour maintenir l'ordre, arrêter les délinquants et faire exécuter les décisions des tribunaux, les seigneurs justiciers n'ont pas de personnel spécifique à leur disposition et doivent utiliser leur sergent, tandis qu'au niveau provincial, la maréchaussée est chargée par la monarchie du maintien de l'ordre, mais avec des effectifs très faibles: au milieu du XVIII ${ }^{e}$ siècle, à l'apogée de sa très relative puissance, elle comprend à l'échelle du royaume 33 prévôts généraux, 111 lieutenants, 151 maréchaux des logis, 732 brigadiers et 2650 hommes, pour une population de 25 millions d'habitants ${ }^{17}$, de sorte que tout délinquant qui quitte rapidement les lieux de son crime (pour autant qu'il ait été dénoncé!) est quasiment assuré de l'impunité. En ville, la police ne devient plus efficace que la maréchaussée rurale qu'à partir de la fin du XVII ${ }^{e}$ siècle, avec la création de charges de lieutenants de police dans les grandes villes; si l'organisation policière repose au début du XVIII ${ }^{\mathrm{e}}$ siècle sur deux ou trois institutions selon les lieux, les polices bourgeoises, héritières des milices médiévales, les polices municipales, composées de sergents salariés très peu nombreux, enfin dans les villes de garnison (c'est-à-dire surtout dans le Nord et l'Est), la police militaire, compétente aussi envers les civils ${ }^{18}$, l'efficacité de l'ensemble reste limitée, et pour les délinquants habituels la fuite continue souvent à assurer l'impunité. La situation n'est pas anarchique pour autant: il faut insister sur l'importance de l'autosurveillance des populations, tant à la ville qu'à la campagne, laquelle permet le plus souvent d'éviter d'entrer dans des situations conflictuelles ou d'atténuer les conséquences de celles-ci. Mais si le besoin surgit, pour telle ou telle raison, de faire appel à des forces de police, la possibilité de le faire est très réduite à la campagne et reste relativement limitée en ville.

La justice, elle non plus, ne peut pas régler toutes les sortes de conflits. Les tribunaux royaux étant établis seulement dans les villes, dans des provinces comme la Bourgogne, l'île-de-France et la Normandie, par exemple, il faut souvent parcourir trente kilomètres pour se rendre à un tribunal de bailliage, et cette distance peut être doublée, triplée ou quadruplée dans des régions du Massif Central, comme les parties montagneuses de l'Auvergne, du Velay ou du Gévaudan, où il est de plus impossible de voyager pendant plusieurs mois de l'année. Dans les campagnes, qui

16 Ce peut être le cas des curés quand ils sont présents depuis peu de temps dans une communauté; voir Garnot (1993).

17

Castan, N. (1976, p. 347).

18

Clémens-Denys (1996). 
rassemblent plus des quatre-cinquièmes de la population, c'est aux justices seigneuriales que revient normalement la responsabilité d'engager les premières poursuites au criminel; or, bien que présentes partout dans le royaume et proches des justiciables, et malgré une activité généralement importante en ce qui concerne les affaires civiles, la plupart répugnent à engager des poursuites dans les affaires criminelles parce qu'elles en seraient rapidement dessaisies par la justice royale compétente (le bailliage ou la sénéchaussée le plus proche) et qu'elles n'en tireraient aucun avantage pécuniaire, seulement des frais... sans parler bien sûr de la pression de l'opinion locale qui pousse, nous l'avons vu, à la «discrétion» vis-à-vis de la justice royale.

Bien plus, même lorsqu'elle en a connaissance, la justice a tendance à limiter d'elle-même volontairement son immixtion dans les affaires internes des communautés locales, soit parce qu'elle juge les différends de peu d'intérêt lorsqu'ils ne portent pas vraiment atteinte à l'ordre public (elle poursuit alors uniquement les délits qui lui paraissent graves), soit parce qu'elle craint d'être submergée par un trop grand nombre de procès; si bien qu'on a pu avancer l'idée que pendant l'Ancien Régime, la justice pénale se consacrait essentiellement au maintien de l'ordre public (tous les «cas royaux», en particulier les assassinats, séditions, crimes de lèsemajesté divine et humaine, etc.), tandis que les communautés assuraient ellesmêmes la fonction de censure des comportements déviants qui ne menacent pas celui-ci (violences verbales, petites violences physiques, atteintes à la réputation et à l'honneur, etc.). Dans les sénéchaussées de Libourne et de Bazas, par exemple, de 1696 à 1789 , les procureurs du roi ne poursuivent que les homicides, les infractions religieuses, et une fois sur deux seulement les infractions à l'ordre public comme le vagabondage et la désertion, mais ils ignorent les autres infractions, y compris les vols ${ }^{19}$. En réalité, le partage des tâches entre la justice et les communautés est beaucoup plus ambigu qu'il n'y paraît au premier regard: la distinction tranchée faite un peu plus haut ne tient compte que du contentieux, c'est-à-dire de la criminalité poursuivie, mais pas de la multitude des cas pourtant susceptibles d'être jugés comme «royaux» et qui ne parviennent jamais en justice parce que la population ne les y porte pas... La situation est donc complexe et nuancée, variant pour chaque type d'affaire en fonction de la conjoncture locale et de l'époque prise en compte (la justice s'intéresse davantage à la répression du vol au XVIII ${ }^{\mathrm{e}}$ siècle qu'au XVII ${ }^{\mathrm{e}}$ siècle, par exemple), et aussi des milieux sociaux concernés (les «étrangers " au village sont bien davantage susceptibles de poursuites pénales que les gens du crû, quel que soit le type de crime dont on les accuse): on voit ainsi alterner, se succéder, coexister aussi parfois, des situations de complémentarité et des situations de concurrence entre le contrôle social effectué par la justice et celui qui relève des communautés. On pourrait faire des observations similaires à propos des forces de police: en matière de braconnage, par exemple, les libertés prises vis-à-vis de la loi sont facilitées par la possibilité d'accommodements quotidiens avec les gardeschasse... ${ }^{20}$, mais au jour le jour, tout dépend finalement des rapports de force locaux. Au total, on doit pourtant bien constater que justice et police participent elles-mêmes en partie à leur relative exclusion du traitement des affaires criminelles, même si, pour l'essentiel, cette exclusion a d'autres origines.

19 Ruff (1984).

20 Salvadori (1996). 
Il ne faut donc accorder qu'une médiocre confiance au fait que les plaignants expliquent souvent leur réticence à porter plainte par l'éloignement excessif du siège de justice. Le non-recours à la justice dans la plupart des affaires criminelles ne résulte pas non plus de l'ignorance de son existence ou de ses mécanismes, mais bien d'une volonté des justiciables, puisque dans les affaires civiles, lorsqu'il y va de leur intérêt, les habitants non seulement des villes, mais aussi des campagnes, n'hésitent pas à engager des procédures en grand nombre et à les poursuivre pendant de nombreuses années ${ }^{21}$. Ce n'est donc ni le mailliage judiciaire, ni la faiblesse de la police, qu'il faut principalement incriminer pour expliquer le faible taux des poursuites par rapport à la criminalité réelle, mais les habitudes et les mentalités. Comment expliquer, par exemple, que les archives judiciaires du XVIII ${ }^{e}$ siècle comportent aussi peu de références à la prostitution, à la sodomie, à la bestialité, aux avortements, sinon par le rôle essentiel que jouent les communautés et les individus dans la sélection des cas qui sont portés devant les tribunaux? Si la justice n'est pas globalement refusée, elle n'est choisie par la population pour le traitement des affaires criminelles que dans des circonstances très précises et largement minoritaires, et faute de la collaboration de la population, la justice ne peut guère influer sur cette situation.

\section{2. - LES CARACTÈRES DE L'INFRAJUSTICE}

Une partie de ce qui échappe à la justice est traitée par l'infrajustice. Encore fautil définir celle-ci ! On peut considérer en premier lieu qu'elle peut concerner tous les types de conflits que la justice est susceptible de traiter, au pénal bien sûr, mais aussi au civil, bref tout les conflits qui peuvent opposer entre eux les individus. En second lieu, l'infrajustice suppose une certaine organisation qui repose sur un consensus social, au moins au niveau local: en particulier l'intervention de tiers, individuels ou collectifs, pour parvenir à un règlement entre les parties ou pour l'entériner, règlement qui a concrètement force d'obligation morale et sociale aux yeux non seulement des parties, mais de tous les membres de la communauté concernée; l'infrajustice a donc un caractère public ou semi-public, parfois même officiel (lorsqu'elle implique un notaire, par exemple, ou une institution). C'est dire, en troisième lieu, que les affaires qui bénéficient de règlements privés n'appartiennent pas au domaine de l'infrajustice.

\section{A. Le rôle du milieu}

L'initiative d'un processus infrajudiciaire peut être prise soit par le milieu, qui cherche par le biais d'un médiateur à résoudre un conflit afin de conserver la cohérence du groupe, soit par la victime, soit par le coupable. Le déclenchement du processus implique ensuite tout un rituel (ce qui met bien en valeur son caractère public et reconnu par la communauté) à l'initiative du coupable. Ainsi le coupable d'une violence, pour marquer préalablement la reconnaissance de sa culpabilité et sa volonté de réparer les conséquences de son acte, peut faire savoir à la famille de sa victime que la facture du chirurgien qui la soigne, ou celle du boucher qui fournit la

21 Billacois (1990). 
viande nécessaire aux bouillons réputés efficaces en cas de blessure, sont à sa charge. Mais encore faut-il que s'enclenche ce processus!

Il est bien évident que si une telle démarche peut être parfois spontanée, elle ne l'est pas dans la plupart des cas; pour la déclencher, des pressions sont le plus souvent nécessaires, et c'est le milieu qui les exerce sous des formes qui peuvent être diverses: il n'y a pas d'infrajustice sans intervention du milieu ni sans publicité. L'une des formes de pression est l'exercice de la violence, physique ou verbale (ou les deux à la fois); les quolibets, les injures, les charivaris, les coups mêmes, peuvent être des modes de pression permettant un règlement pacifique ultérieur. Ainsi il n'est pas rare de voir un coupable arrêté par les habitants eux-mêmes pour subir toutes sortes de brimades physiques et morales après une décision prise collectivement en sa présence par les habitants, fréquemment avec la participation et même sous la direction des notables, curé compris (c'est par exemple souvent les cas des voleurs d'animaux, chevaux ou bovins, dans la Bretagne du XVIII ${ }^{e}$ siècle ${ }^{22}$ ), tout cela pour le forcer à engager une procédure infrajudiciaire de restitution des biens volés ou de dédommagement de ses victimes.

Mais le plus souvent, les pressions préalables exercées sur la partie coupable sont d'une nature plus pacifique. Les curés peuvent y jouer un rôle essentiel, puisqu'ils sont susceptibles, la crainte de l'enfer aidant, de suggérer fortement des restitutions d'objets volés ou de négocier des dommages et intérêts; par le biais de la confession, qui constitue aussi une forme d'aveu, les curés peuvent également favoriser de futurs arrangements par une pression discrète ${ }^{23}$. Il est fréquent également que la victime engage une procédure judiciaire pour faire pression sur son adversaire et le forcer à composer sur le plan infrajudiciaire (car de toute manière, il lui en coûterait toujours moins cher par ce dernier moyen que par le premier). Vers 16471657, dans la justice seigneuriale de Saint-Germain-en-Laye, les trois quarts des affaires d'injures et de brutalités sans homicide ne dépassent pas le stade de la plainte ou de l'information: on retrouve de nombreux accords passés devant notaire (essentiellement pour insultes et horions) moins d'une semaine après les faits, à un stade précoce de la procédure judiciaire, qui s'arrête alors ${ }^{24}$. En Bourgogne au $\mathrm{XVIII}^{\mathrm{e}}$ siècle, la majorité des procédures s'arrête aussi avant son terme, et pour un grand nombre d'entre elles on retrouve des accommodements signés devant notaire ${ }^{25}$; il en va de même en Bretagne ${ }^{26}$.

Justice et infrajustice peuvent donc se mélanger ou se succéder dans la chronologie d'une affaire, ce qui signifie qu'il n'y a pas en général de conflit entre l'une et l'autre, plutôt une complémentarité; on pourrait même considérer que l'infrajustice découle de l'existence même d'une justice permettant éventuellement la résolution du délit. De sorte que tout compte fait, si le recours à la justice est minoritaire pour traiter les affaires criminelles, lorsqu'il est quand même utilisé, c'est dans la majorité des cas pour l'abandonner plus ou moins rapidement et régler finalement les affrontements concernés loin du regard des juges.

\footnotetext{
22 Quéniart (1993).

23 Quéniart (1996).

24 Isbled (1990).

25 Girardot-Clément (1995).

26

Mer (1985).
} 


\section{B. Les médiateurs-arbitres de l'infrajustice}

Il n'est pas d'infrajustice sans intervention d'un tiers, individuel ou collectif, souvent pour déclencher le processus infrajudiciaire, ensuite soit pour entériner l'accord auquel les parties arrivent d'elles-mêmes (dans ce cas, il n'intervient qu'en fin de négociation), soit pour arbitrer et décider d'un accommodement (dans ce cas, il intervient dès le début ou au cours de la négociation). La liste de ces tiers, qu'on peut appeler les «médiateurs-arbitres » de l'infrajustice, est longue et variée: notables divers (en particulier les notaires), curés chez les catholiques et consistoires chez les protestants ${ }^{27}$, seigneurs et syndics de communautés dans les campagnes, échevins et maires dans les villes, groupes de jeunesse, associations professionnelles, bureaux des hôpitaux, etc., mais aussi membres de l'institution judiciaire ou policière lorsqu'ils agissent non en tant que juges, mais en tant qu'arbitres (c'est souvent le cas des commissaires de police à Paris au XVIII ${ }^{\mathrm{e}}$ siècle $^{28}$ ); sans parler de n'importe quel individu, qui peut être pris un jour ou l'autre comme arbitre pour régler telle ou telle affaire (tout est parfois affaire de circonstances: dans un cabaret, c'est le patron qui peut occasionnellement être amené à jouer ce rôle, par exemple). Il peut donc y avoir une certaine liberté de choix pour les parties, en fonction du type d'affaire, des compétences respectives des médiateurs-arbitres présents localement, et aussi sans doute des affinités personnelles.

Ces médiateurs-arbitres de l'infrajustice (parfois seulement médiateurs, parfois seulement arbitres, parfois les deux à la fois) exercent une sorte de magistrature d'influence, grâce à des compétences particulières venant de leurs études, de leurs pratiques professionnelles, de leur âge, de leur prestige... Parmi eux, les renseignements archivistiques disponibles privilégient les notaires pour des raisons documentaires évidentes; ainsi à Paris, les règlements devant notaire du contentieux criminel sont surtout pratiqués dans la classe moyenne ou artisanale, alors que les riches peuvent défrayer une poursuite en justice et que les pauvres s'efforcent d'éviter les droits notariaux par des règlements conclus devant témoins sans caractère officiel $^{29}$ (ce qui, au passage, pose le problème des critères sociaux dans le choix de la justice ou de l'infrajustice). Quant aux curés, ils peuvent court-circuiter une procédure judiciaire lorsque des monitoires ont été décidés par la justice et que par voie de conséquence ils apprennent la vérité sur une affaire, qu'ils cherchent alors à règler eux-mêmes sans en référer au magistrat instructeur ${ }^{30}$. Certains médiateursarbitres sont même institutionnalisés, comme les «apaiseurs » établis depuis longtemps dans chaque quartier des villes du Nord ${ }^{31}$. Mais pour ces derniers, on peut se demander s'ils n'appartiennent pas davantage à la justice qu'à l'infrajustice... observation qui a au moins le mérite de montrer, encore une fois, qu'un fossé infranchissable ne sépare pas l'une et l'autre. Le prouve aussi le fait que les médiateursarbitres, quels qu'ils soient, peuvent aussi de par leur autorité morale décider de traiter une affaire localement ou de la porter en justice, ce qui ajoute à leur rôle de rouages essentiels de l'infrajustice celui de pivots entre celle-ci et la justice. En

\footnotetext{
27 Poton (1992).

28 Dinges (1996).

29 Soman (1982).

30 Quéniart (1996).

31 Clémens-Denys (1996).
} 
outre, le rôle de ces médiateurs-arbitres de l'infrajustice ne se limite pas à celle-ci ; il se manifeste également lors d'autres moments tout aussi importants de la vie sociale, comme la conclusion des mariages ou encore les partages successoraux.

\section{Les modes de règlement de l'infrajustice}

Alors que la justice condamne (ou acquitte), l'infrajustice a pour but (outre d'éviter la justice) de rétablir l'entente, ce qui constitue une différence essentielle. Elle le fait par le biais de modes de règlement qui n'aboutissent jamais à une exclusion, mais qui cherchent seulement à rétablir un équilibre social momentanément brisé : soit une transaction (elle suppose un accord proposé par un tiers ou décidé par les parties elles-mêmes), soit un arbitrage (la décision est alors prise par ce tiers et doit s'imposer aux parties).

Qu'ils découlent de transactions ou d'arbitrages, et quels que soient les épisodes préalables qui y mènent, ou trouve deux principales catégories de règlements, qui ne sont pas forcément exclusives l'une de l'autre: les «réparations d'honneur» et les compensations financières, chacune pouvant impliquer un reconnaissante écrite, en général devant notaire, ou orale (une réconciliation en public, par exemple, devant des témoins, avec des gestes ritualisés, comme le baiser, le serrement de mains, et des engagements précis). Les réparations d'honneur sont quasiment institutionnalisées dans de nombreuses régions, qu'il s'agisse par exemple des villes du Nord où les «apaiseurs» sont chargés d'aider les parties à y parvenir ${ }^{32}$, ou encore des communautés bourguignonnes, où le rituel de ces réparations est codifié précisément (la justice peut d'ailleurs décider aussi des cérémonies similaires). Quant à l'importance des compensations financières, elle dépend de la nature de l'offense, de la qualité de l'offensé et du fautif, de l'entourage de la victime, éventuellement du nombre et de l'importance sociale des témoins. Au total, qu'il s'agisse de réparations d'honneur ou de compensations financières, ou des deux ensemble, il s'agit toujours de dédommager la victime et sa famille, donc de rétablir un équilibre qui a été momentanément détruit par une transgression, et de le faire d'une manière qui paraît à chacun beaucoup plus sûre que celle que la justice pourrait décider: les compensations financières sont toujours adaptées aux capacités du débiteur, les réparations d'honneur sont effectuées localement devant tous les individus concernés, témoins compris. On peut aussi trouver une variante de ces pratiques, le "pardon »: il est attesté en Roussillon sous la forme d'un acte notarié qui est réalisé à l'initiative de l'offensé et qui, intervenant au début d'une action judiciaire, l'interrompt définitivement ${ }^{33}$; il est probable que cette pratique existe aussi dans d'autres provinces.

\section{3. - HORS DE LA JUSTICE ET DE L'INFRAJUSTICE: PARAJUSTICE ET EXTRAJUSTICE}

L'infrajustice n'est pourtant pas une panacée. Bien qu'elle traite sans doute davantage d'affaires que la justice elle-même, elle ne concerne pas tous les écarts aux normes qui ne sont pas réglés par celle-ci, il s'en faut de beaucoup. Même si ce

\footnotetext{
32 Clémens-Denys (1996).

33 Larguier (1996).
} 
terrain de recherche est encore insuffisamment balisé, il semble évident qu'une part importante de la criminalité est traitée en marge de la justice et de l'infrajustice (c'est pourquoi je l'appellerai la "parajustice»), et qu'une autre ne connaît aucune tentative de règlement, reste subie ou tolérée, et se situe ainsi hors de la justice (c'est pourquoi je l'appellerai l' «extrajustice»).

\section{A. La parajustice: des modes de règlement privés ou collectifs}

La justice et l'infrajustice supposent la publicité, tandis que la parajustice se caractérise par des procédures privées, sans intervention active d'un tiers, qui se concrétisent soit par des arrangements soit par des vengeances, lesquels ont finalement les uns et les autres le même but, qui est de rétablir un équilibre social préalablement brisé par une transgression (comme l'infrajustice, par conséquent, mais par des moyens différents). On n'insistera guère ici sur les arrangements privés, qui se négocient, par définition, sans aucune intervention extérieure aux parties (faute de quoi ils relèveraient de l'infrajustice): oraux presque toujours, ils n'ont guère laissé de traces archivistiques, sinon dans des correspondances et dans des mémoires, ce qui limite leur connaissance par les historiens pour l'essentiel aux catégories sociales dominantes ${ }^{34}$, mais ne signifie pas pour autant qu'ils ne concernent pas toutes les autres catégories sociales. Leur nombre est certainement très important, peut-être même davantage que celui des affaires qui bénéficient d'un règlement infrajudiciaire.

La criminalité ambiante peut aussi entraîner des réactions de vengeance, qui génèrent elles-mêmes une autre forme de criminalité (qui peut un jour d'ailleurs passer en justice ou en infrajustice, ou rester subie ou tolérée, donc dans le domaine de l'extrajustice). Condamnables en justice, intolérables en infrajustice (puisqu'il ne s'agit pas dans ce cas de rétablir l'entente, tout au contraire), elles participent elles aussi, à leur place, à l'organisation de la régulation sociale: la vengeance, qu'elle ait pour forme l'injure, la diffamation, les coups et blessures (jusqu'au meurtre) ou les atteintes aux biens, a pour finalité de rétablir l'équilibre et la paix sociale au sein de la communauté, au même titre, mais par des moyens différents, que la justice et que l'infrajustice. On connaît l'importance des vengeances privées (individuelles ou familiales) dans les sociétés pré-modernes, mais on a sans doute sous-estimé leur pérennité dans la France d'Ancien Régime, au profit des règlements judiciaires et infrajudiciaires, beaucoup mieux représentés dans les sources et par là surévalués; ce n'est pas parce que la législation les interdit que ces pratiques n'ont pas perduré, et peut-être même augmenté. Le duel constitue un bon exemple de cette réalité; bien qu'interdit légalement au cours de la période (alors qu'il avait encore une valeur judiciaire au début du $\mathrm{XVI}^{\mathrm{e}}$ siècle, il sort ensuite du domaine judiciaire pour entrer dans le domaine parajudiciaire...), sa pratique perdure pendant tout l'Ancien Régime dans tous les milieux, même si ses caractéristiques se modifient ${ }^{35}$, parce qu'elle est socialement nécessaire: le duelliste, s'il est un criminel aux yeux des lois (ce qui est secondaire...) lorsqu'il sauve sa réputation l'arme à la main, passerait pour un lâche aux yeux du milieu (ce qui est essentiel...) s'il respectait la loi prohibant la violence comme mode de règlement des litiges privés ${ }^{36}$, de sorte que l'obli-

34 Desaive (1996).

35 Billacois (1986).

36 Porret (1996, p. 184). 
gation sociale de défense de l'honneur l'emporte le plus souvent sur l'interdiction légale de la violence privée. Or le duel n'est qu'une des modalités du système vindicatoire, la mieux connue certainement, parmi d'autres encore très mal connues, au sein desquelles figurent notamment les représailles morales et psychologiques qu'il faudrait se résoudre à étudier sérieusement un jour... Des études régionales ont d'ailleurs montré la prégnance de ce système vindicatoire au XIX ${ }^{e}$ siècle encore (et même après), non seulement dans des régions rurales enclavées, comme les Pyrénées ${ }^{37}$, mais aussi dans des régions proches de Paris, comme l'arrondissement de Rambouillet ${ }^{38}$ (on sait par ailleurs qu'en Allemagne, par exemple, le duel était en recrudescence au XIX ${ }^{\mathrm{e}}$ siècle). On gagnerait donc à s'intéresser de plus près à ces pratiques dans la France d'Ancien Régime, non seulement en examinant leurs diverses modalités pratiques, mais aussi en cherchant à vérifier si elles ne concernent que les réactions à des délits précis, essentiellement les affaires d'honneur, ou à tous les délits indifféremment.

Les réactions collectives de rejet sont mieux connues que les vengeances individuelles ou familiales, sans doute parce qu'étant plus spectaculaires, elles ont laissé dans les documents des traces plus facilement lisibles. Si elles supposent une évidente publicité, elles ne revêtent, à la différence des modalités de l'infrajustice, aucun caractère officiel ou semi-officiel. On voit ainsi des communautés villageoises entières conduire des expéditions punitives contre des villages voisins ayant porté atteinte à leurs intérêts, ainsi que des nombreuses bagarres entre groupes de jeunesse issus de villages proches, lesquels défendent tout autant l'honneur de leur village que les droits qu'ils s'octroient sur le «stock» des filles à marier. Ces réalités-là sont déjà bien analysées ${ }^{39}$. On connaît moins les règlements de comptes collectifs exercés par une communauté unanime sur l'un de ses membres. Un bon exemple est constitué par cette affaire spectaculaire survenue à Palinges en Charolais au début du XVIII ${ }^{e}$ siècle, où les villageois, en conflit avec leur curé accusé de "violences qu'il commet journellement, ne parlant que de tuer et assommer ceux qui l'approchaient » et d'injurier ses paroissiens qu'il traite de «bougre de charogne, bougre de coquin, bougre de putain », règlent eux-mêmes le problème en l'assassinant: dans cette affaire, le meurtrier n'est pas un marginal ou un étranger, mais la communauté dans son ensemble, même si l'acte est commis par quelques hommes seulement, mais avec l'assentiment de la population locale ${ }^{40}$. On conviendra que ces manières d'agir se situent bien loin à la fois de la justice et de l'infrajustice, et qu'elles constituent par elles-mêmes une forme de criminalité aux yeux de la loi; pour autant, elles ne doivent pas être perçues comme des manifestations de désordre, mais au contraire comme un mode, parmi d'autres, de régulation sociale, au même titre que la justice et que l'infrajustice. Comme cette dernière, la parajustice sert à éviter l'intervention de la justice, mais elle le fait sans intervention extérieure, sans témoin et sans publicité.

\footnotetext{
37 Soulet (1987).

38 Chauvaud (1995).

39 Muchembled (1989).

40 Barbier (1994).
} 


\section{B. L'extrajustice: une criminalité subie ou tolérée}

L'extrajustice est le seul mode d'appréhension de la criminalité qui ne débouche sur aucun règlement de celle-ci; à la différence de la justice, de l'infrajustice et de la parajustice, elle ne cherche pas à résoudre des conflits. Cette situation d'extrajustice se décline sur deux modes: celui de la criminalité subie contre leur gré par les habitants, et celui de la criminalité qu'ils tolèrent volontairement.

Si l'on subit sans réaction une partie de la criminalité, c'est pour deux catégories de raisons. La première raison tient à la force des choses, ou, en d'autres termes, aux nécessités de la vie en communauté. On est bien forcé de subir sans réagir une partie des déviances commises par autrui dans une société de voisinage où la curiosité mutuelle est omniprésente: chacun est souvent obligé par les circonstances de la vie, non seulement de connaitre, mais encore de supporter le colérique, le violent, l'impie, le blasphémateur ou le provocateur qui vit dans son environnement, ne serait-ce que pour faire supporter lui-même ses propres déviances. Dans une société fermée (un village, une petite ville sans «étrangers»), tout le monde connaît généralement les coupables des délits, mais sait aussi que la dénonciation de l'un d'entre eux, que ce soit sous des formes judiciaires ou infrajudiciaires, risquerait d'entrainer des réactions en chaîne qui ne l'épargneraient pas, lui non plus. On subit ainsi les autres pour que les autres vous subissent...

La seconde cause de l'existence d'une criminalité subie sans réaction, la plus importante peut-être, est la crainte qu'inspirent à leurs voisins certains délinquants, que ce soit pour des raisons sociales (quand ce sont des notables, ou le seigneur) ou pour des raisons physiques (quand il s'agit de bandes, ou simplement d'un individu brutal): la crainte des représailles physiques ou économiques impose la retenue, donc la patience... On se rend bien compte de l'existence de ces individus, tellement craints qu'on est forcé de subir leurs crimes sans réagir, à l'occasion par exemple des Grand Jours ${ }^{41}$ qui se tiennent épisodiquement aux XVI ${ }^{e}$ et XVII ${ }^{e}$ siècles, et qui sont bien connus pour le Poitou en 1634 et pour l'Auvergne en $1665^{42}$. On s'aperçoit à ces occasions qu'existe fréquemment tout un monde de non-droit, où règne la loi du plus fort, et que ni la justice, ni l'infrajustice, ni la parajustice, ne parviennent à éradiquer, puisqu'elles ne peuvent véritablement fonctionner que lorsque la société concernée est à peu près correctement régulée, c'est-à-dire lorsque les pouvoirs ne sont exercés par aucun individu ou aucun groupe de façon trop hégémonique. Les Grands Jours fournissent des exemples spectaculaires de ce genre de situation, mais les archives judiciaires ordinaires montrent parfois, en négatif, que des situations similaires, aux degrés de gravité très divers selon les lieux et les époques, peuvent être constatées aussi un peu partout. Il suffit par exemple de songer à la crainte suscitée dans les campagnes par les brigands et par les bandes de vagabonds, crainte encore sensible et même en recrudescence à la veille de la Révolution, pour comprendre que la loi du plus fort peut s'appliquer ici ou là, plus ou moins longtemps, et que ceux qui en bénéficient peuvent échapper à toute régulation judiciaire ou infrajudiciaire.

Une autre partie de la criminalité est tolérée volontairement par les communautés, non sans qu'elles exercent sur elle une certaine surveillance et que cette tolé-

41 Sessions extraordinaires tenues par les parlements en dehors de leur siège ordinaire, dans des villes éloignées de celui-cí, afin d'offrir un recours commode aux justiciables et de réparer les abus.

42

Cornette (1998); Lebigre (1976). 
rance puisse rencontrer des limites. On a ainsi l'habitude d'admettre sans réaction la petite violence quotidienne, banale, ordinaire: nul ne songe à reprocher aux mâ̂tresses de maisons de gifler leurs servantes, ni aux artisans de rudoyer leurs apprentis $^{43}$. Quant à la violence épisodique, plus rare et plus dangereuse, elle est presque aussi bien tolérée: les rixes ne font pas souvent l'objet de poursuites ni de règlements à l'amiable (sauf en cas de débordements), surtout lorsqu'elles se produisent au cabaret ou les soirs de fêtes, parce qu'elles apparaissent aux habitants comme des occasions de défoulement pour la jeunesse, fréquemment même comme des nécessités lorsqu'il s'agit de défendre l'honneur du village, du quartier ou du métier ${ }^{44}$. Il est évident que dans ce domaine aussi joue à plein le décalage entre la loi d'une part, les habitudes sociales d'autre part.

Les communautés tolèrent également sans réactions l'existence d'individus qui vivent, au moins en partie, de manière permanente cette fois, d'illégalismes; il s'agit pour l'essentiel de marginaux, mais des marginaux en général originaires du lieu et partiellement intégrés dans la communauté, tout en se distinguant de l'immense majorité de ses membres par la non-conformité au moins partielle de leur manière de vivre. Si leurs différences et leurs déviances sont tolérées, c'est d'une part parce qu'il peut être utile à toute communauté de conserver en son sein un espace de marginalité pour souder par contraste sa propre cohérence, d'autre part parce que les activités illégales de ces marginaux sont utilisées à leur profit par les habitants qui n'hésitent pas, par exemple, à consulter les sorciers ou à acheter du gibier aux braconniers. C'est conjoncturellement, s'il se produit un événement qui ébranle la cohésion de la communauté et qui paraît la menacer, que ces marginaux risquent d'en devenir, même sans preuves, les responsables putatifs aux yeux d'une partie ou de la quasi-totalité des habitants: de marginaux tolérés, voire intégrés, ils sont alors transformés en criminels rejetés par leurs pairs et éventuellement livrés à la justice ou forcés de passer par les étapes rituelles de l'infrajustice ${ }^{45}$ (ou encore par les modalités de la parajustice). Mais au lieu de considérer que ces individus finissent forcément par tous passer en justice ou en infrajustice parce qu'ils réussiraient à lasser un jour la tolérance de leurs compatriotes, il serait sans doute plus exact d'inverser la réflexion et de considérer que la tolérance reste la règle largement majoritaire, le passage devant la justice ou l'infrajustice (ou les deux) l'exception.

Le stade ultime de la criminalité tolérée est atteint lorsque la communauté tout entière en devient elle-même l'acteur. Les habitants, en effet, s'accordent généralement pour échapper aux conséquences fâcheuses d'actes qu'ils estiment sans dommage pour autrui à l'intérieur de leur communauté, bien que ces actes soient illégaux, d'où une dissimulation collective systématique dans les domaines concernés, notamment la fraude fiscale, la contrebande, le braconnage ${ }^{46}$ (à l'inverse, lorsque la communauté se sent menacée matériellement ou physiquement, elle n'hésite pas à faire appel à la justice, comme lors des vols de pièces du mobilier destiné au culte, lesquels appartiennent directement à la communauté villageoise par le biais de la fabrique, et dans toutes les affaires où il est question de cimetière paroissial ${ }^{47}$ ).

44 Muchembled (1989).

45 Garnot (1999).

46 Brunet (1986); Ferrer (1998); Salvadori (1996).

47 Dyonet (1990). 
Dans ces cas, il s'agit bien d'une forme de criminalité tolérée, et qui l'est d'autant plus que chaque membre de la communauté en est à la fois un spectateur volontairement passif et un acteur.

Dans la France moderne, l'infrajustice ne constitue nullement une sous-justice, mais un mode original, souple et divers, de règlement des conflits, tout aussi efficace que la justice, voire bien davantage. Mais pour autant, elle ne concerne pas, il s'en faut de beaucoup, tous les comportements délictueux inconnus de la justice, dont beaucoup restent étrangers à tout mode de règlement, ou encore sont réglés par des modalités privées. Il ne faudrait donc pas tomber dans l'excès qui consisterait à considérer qu'en matière de criminalité tout ce qui ne passe pas en justice dans la France moderne relève de l'infrajustice, même si beaucoup en relève ${ }^{48}$, faute de quoi on tomberait dans une nouvelle illusion historiographique en faisant de l'infrajustice une sorte de «fourre-tout». En réalité, il existe une large variété de modes de traitement des conflits, dans lesquels s'insèrent, mais seulement parmi d'autres, justice et infrajustice, et qui offrent ainsi aux individus et aux groupes une vaste gamme d'options et de stratégies.

Dans cette perspective, les recherches à venir pourraient se diriger vers deux directions. D'une part, il faudrait chercher à mieux connaître tout ce qui se situe hors de la justice et de l'infrajustice, en en faisant un objet de recherche spécifique: après l'étude du contentieux, puis celle de l'infrajustice, voilà une troisième étape à franchir dans l'étude de la criminalité en France à l'époque moderne. Les documents permettent ce genre d'étude, puisque nombre d'affaires qui passent en justice, soit mettent en scène incidemment des épisodes antérieurs de ce type, soit en sont même directement la conséquence, tandis que des procédures exceptionnelles permettent aussi d'en révéler un certain nombre.

Pour autant, il ne saurait être question d'abandonner l'étude de l'infrajustice, sous prétexte que son importance a été exagérée. Si ses modalités techniques semblent aujourd'hui assez bien connues, en effet, il n'en va pas de même de sa chronologie ni de sa géographie. La période moderne peut apparaître comme un tournant dans l'histoire de l'infrajustice, dans la mesure où elle voit la montée du pouvoir de l'État qui cherche à imposer à la population les institutions judiciaires, de sorte qu'on pourrait en conclure à un recul de l'infrajustice, comme le prouverait le déclin de la pratique des règlements infrajudiciaires devant notaire à Paris au XVIII siècle ${ }^{49}$. Mais plutôt que de recul, ne faudrait-il pas parler de changement de compétences, l'infrajustice ne constituant aucunement une série de pratiques résiduelles, mais agissant en interaction avec les pratiques judiciaires? Ainsi, la baisse relative du contentieux de la violence au cours du XVIII ${ }^{\mathrm{e}}$ siècle pourrait s'expliquer par une plus grande prise en compte de celle-ci par l'infrajustice ${ }^{50}$. Quant à la géographie de l'infrajustice, elle mériterait aussi d'être examinée de plus près; on peut supposer que son emprise est d'autant plus importante qu'une région ou une communauté est éloignée des instances officielles de régulation sociale ${ }^{51}$, mais cette hypothèse demande encore à être confirmée. En outre, l'examen détaillé des compensations

\footnotetext{
48 Garnot (1996).

49 Soman (1982).

so Dinges (1996).

51 Cameron (1981).
} 
financières décidées par les médiateurs-arbitres de l'infrajustice permettrait de révéler les différences entre les normes et les valeurs de la société locale, et celles exprimées dans les lois et dans la jurisprudence.

Plutôt que de placer tout, à tort et à travers, dans l'infrajustice, cherchons plutôt à en préciser les détails et les évolutions. Nous pourrons aussi, en même temps, relativiser son importance réelle au bénéfice de l'analyse des pratiques qui ne se situent ni dans son champ, ni dans celui de la justice.

\author{
Benoît GARNOT \\ Université de Bourgogne/France \\ UMR CNRS 5605 \\ 2, boulevard Gabriel \\ 21000 Dijon \\ E-mail: benoit.garnot@wanadoo.fr
}

\title{
Bibliographie
}

Barbier, S., Les mentalités dans un village du Charolais à travers une affaire criminelle au XVIII siècle, mémoire de maîtrise dactylographié, Dijon, 1994.

Billacois, F., Le duel dans la société française des XVI et XVII siècles, Paris, Éditions de l'École des hautes études en sciences sociales, 1986.

Billacois, F. et alii, Porter plainte: stratégies villageoises et institutions judiciaires en $\hat{l} l e-d e-$ France (XVII'-XVIII' ${ }^{e}$ siecles)», Droit et cultures, 1990, 19, pp. 7-148.

Brunet, M., Le Roussillon. Une société contre l'État. 1780-1820, Toulouse, Association des publications de l'université de Toulouse-Le Mirail, Eché, 1986.

Cameron, I.A., Crime and Repression in the Auvergne and the Guyenne, 1720-1790, Cambridge, Cambridge University Press, 1981.

Castan, N., La justice expéditive, Annales (Économies Sociétés Civilisations), 1976, 2, pp. 331-361.

Castan, N., Les criminels de Languedoc. Les exigences d'ordre et les voies du ressentiment dans une société prérévolutionnaire (1750-1790), Toulouse, Publications de l'université de Toulouse-Le Mirail, 1977.

Castan, N., Justice et répression en Languedoc à l'époque des Lumières, Paris, Flammarion, 1980.

Castan, N. et Y., Une économie de justice à l'âge moderne, Histoire, Économie et Société, 1982, 3, pp. 361-368.

Castan, Y., Honnêteté et relations sociales en Languedoc, 1715-1780, Paris, Flammarion, 1979.

Chauvaud, F., Les passions villageoises au XIX $X^{e}$ siècle. Les émotions rurales dans les pays de Beauce, du Hurepoix et du Mantois, Paris, Publisud, 1995.

Clémens-Denys, C., Entre justice subalterne et infrajustice, les apaiseurs des Flandres des origines au XVIII ${ }^{e}$ siècle ou la conciliation au service de la cité, in Garnot, B. (dir.), L'infrajudiciaire du Moyen Age à l'époque contemporaine, Dijon, Éditions universitaires de Dijon, 1996, pp. 257-272.

Cornette, J., La mélancolie du pouvoir. Omer Talon et le procès de la raison d'État, Paris, Fayard, 1998. 
Desaive, J.-P., Patrimoine et pressions familiales: sources littéraires et textes d'archives, in Garnot, B., (dir.), L'infrajudiciaire du Moyen Age à l'époque contemporaine, Dijon, Éditions universitaires de Dijon, 1996, pp. 373-382.

Dinges, M., Négocier son honneur dans le peuple parisien au XVIII siècle: la rue, «l'infrajudiciaire » et la justice, in Garnot, B., (dir.), L'infrajudiciaire du Moyen Age à l'époque contemporaine, Dijon, Editions universitaires de Dijon, 1996, pp. 393-404.

Dyonet, N., Impiétés provinciales au XVIII' siècle, Histoire, Économie et Société, 1990, 3, pp. 391-422.

Ferrer, A., La petite contrebande frontalière de la fin du XVIII ${ }^{c}$ siècle. L'exemple comtois, in Garnot, B. (dir.), La petite délinquance du Moyen Age à l'époque contemporaine, Dijon, Éditions universitaires de Dijon, 1998, pp. 341-350.

Figueira, A., Les mentalités et les comportements populaires à Courtivron au XVIII siècle (d'après la justice seigneuriale. 1700-1785), mémoire de maîtrise, dactylographié, Dijon, 1995.

Garnot, B., Une illusion historiographique: justice et criminalité au XVIII ${ }^{e}$ siècle, Revue Historique, $1989, \mathrm{n}^{\circ}$ 570, pp. 361-379.

Garnot, B., Pour une histoire nouvelle de la criminalité au XVIII ${ }^{\mathrm{e}}$ siècle, Revue Historique, 1992,584 , pp. 289-303.

Garnot, B., Un crime conjugal au XVIII siècle. L'affaire Boiveau, Paris, Imago, 1993.

Garnot, B., (dir.), L'infrajudiciaire du Moyen Age à l'époque contemporaine, Dijon, Éditions universitaires de Dijon, 1996.

Garnot, B., Justice, milieu, marginalité et délinquance: un exemple autunois au XVIII ${ }^{e}$ siècle, in Garnot, B., (dir.), De la déviance à la délinquance. $X V^{e}-X X^{e}$ siècles, Dijon, E.U.D., 1999, pp. 117-132.

Girardot-Clément, C., Les justices seigneuriales du bailliage de Châtillon-sur-Seine au XVIIt siècle (études par sondages), mémoire de D.E.A., dactylographié, Dijon, 1995.

Isbled, B., Le recours à la justice à Saint-Germain-des-Prés au milieu du XVII ${ }^{\mathfrak{e}}$ siècle, in Bercé, Y.-M., Castan, Y. (éd.), Les archives du délit, empreintes de société, Toulouse, Éditions universitaires du Sud, 1990, pp. 65-74.

Larguier, G., Pardons catalans, in Garnot, B., (dir.), L'infrajudiciaire du Moyen Age à l'époque contemporaine, Dijon, E.U.D., 1996, pp. 407-417.

Lebigre, A., Les Grands Jours d'Auvergne. Désordres et répression au XVII' siècle, Paris, Hachette, 1976.

Lenman, (B.), Parker, (G.), The State, the Community and the modern law in early modern Europe, in Gatrell, V.A.C., Lenman, B., Parker, G., Crime and the law. The social history in Western Europe since 1500, London, Europa Pubications, 1980, pp. 11-48.

Mantecon, T.A., Conflictividad y discipliniamento social en la Cantabria rural del Antiguo Regimen, Santander, 1997.

Mer, L.-B., La procédure criminelle au XVIII ${ }^{c}$ siècle: l'enseignement des archives bretonnes, Revue historique, 1985, 555, pp. 9-42.

Muchembled, R., La violence au village. Sociabilité et comportements populaires en Artois du XVe au XVIII siècle, Turnhout, Brepols, 1989.

Muchembled, R., Le temps des supplices. De l'obéissance sous les rois absolus. XVe-XVIII siècles, Paris, Armand Colin, 1992.

Porret, M., «Il faut que j'aie ta vie ou que tu aies la mienne»: les «circonstances aggravantes» du duel à Genève au XVIII ${ }^{e}$ siècle ou comment devenir criminel en défendant son honneur, in Garnot, B., (dir.), L'infrajudiciaire du Moyen Age à l'époque contemporaine, Dijon, Éditions universitaires de Dijon, 1996, pp. 175-188.

Porret, M., (éd.), Beccaria et la culture juridique des Lumières, Genève, Droz, 1997. 
Poton, D., Le consistoire protestant au XVII' siècle: un tribunal des mœurs, in Garnot, B. (dir.), Histoire et criminalité de l'Antiquité au $X X^{e}$ siècle. Nouvelles approches, Dijon, Éditions universitaires de Dijon, 1992, pp. 411-417.

Quéniart, J., Le grand Chapelletout. Violence, normes et comportements dans la Bretagne rurale au XVIII' siècle, Rennes, Apogée, 1993.

Quéniart, J., Recteurs et régulation sociale en Bretagne au XVIII siècle, in Garnot, B., (dir.), L'infrajudiciaire du Moyen Age à l'époque contemporaine, Dijon, Éditions universitaires de Dijon, 1996, pp. 231-240.

Rousseaux, X., Lévy, R. (dir.), Le pénal dans tous ses états. Justice, États et sociétés en Europe (XII $-X X^{e}$ siècles), Bruxelles, Publications des Facultés universitaires SaintLouis, 1997.

Ruff, J.-R., Crime, Justice and Public Order in Old Regime France. The Sénéchaussées of Libourne and Bazas, 1696-1789, Londres-Sydney-Dover, Croom Helm, 1984.

Salvadori, P., La chasse sous l'Ancien Régime, Paris, Fayard, 1996.

Shoemaker, R., Procecution and Punishment: Petty Crime and the Law in London and Rural Middelsex, c. 1660-1725, Cambridge, 1991.

Soman, A., L'infrajustice à Paris d'après les archives notariales, Histoire, Économie et Société, 1982, 3, pp. 369-376.

Soulet, J.-F., Les Pyrénées au XIX siècle, Toulouse, Eché, 1987. 